\title{
Composite Gauss-Legendre Formulas for Solving Fuzzy Integration
}

\author{
Xiaobin Guo, ${ }^{1}$ Dequan Shang, ${ }^{2}$ and Xiaoquan $\mathrm{Lu}^{3}$ \\ ${ }^{1}$ College of Mathematics and Statistics, Northwest Normal University, Lanzhou 730070, China \\ ${ }^{2}$ Department of Public Courses, Gansu College of Traditional Chinese Medicine, Lanzhou 730000, China \\ ${ }^{3}$ College of Chemistry and Chemical Engineering, Northwest Normal University, Lanzhou 730070, China
}

Correspondence should be addressed to Xiaoquan Lu; luxq@nwnu.edu.cn

Received 9 December 2013; Revised 6 May 2014; Accepted 6 May 2014; Published 29 May 2014

Academic Editor: Valentina Emilia Balas

Copyright (C) 2014 Xiaobin Guo et al. This is an open access article distributed under the Creative Commons Attribution License, which permits unrestricted use, distribution, and reproduction in any medium, provided the original work is properly cited.

Two numerical integration rules based on composition of Gauss-Legendre formulas for solving integration of fuzzy numbers-valued functions are investigated in this paper. The methods' constructions are presented and the corresponding convergence theorems are shown in detail. Two numerical examples are given to illustrate the proposed algorithms finally.

\section{Introduction}

Numerical integration is one of the basic contents in numerical mathematics, and it always plays a vital role in engineering and science calculation. Numerical integration methods are introduced in detail [1]. Numerical integration is always carried out by mechanical quadrature and its basic scheme [2] is as follows:

$$
\int_{a}^{b} f(x) d x \approx \sum_{k=0}^{n} A_{k} f\left(x_{k}\right),
$$

where $A_{k} \geq 0, k=0,1, \ldots, n$, and $x_{k} \in[a, b], k=0,1, \ldots, n$, are called coefficients and nodes for mechanical quadrature, respectively. Once the coefficients and nodes are set down, the scheme (1) can be determined.

Over years, some works have emerged about the asymptotic properties of numerical integration methods. However, their results are concise, but reasoning processes are very complicated [3-6]. The topic of fuzzy integration was first discussed in [7]. In 2005, Allahviranloo [8] made a good attempt to use Newton Cot's methods with positive coefficients for integration of fuzzy functions. For instance, he designed Trapezoidal integration rule and Simpson integration rule for fuzzy integral. Later, they applied the Gaussian quadrature method and Romberg method for approximation of fuzzy integral and fuzzy multiple integral, built a series of formulas for intricate fuzzy integral [8-11], and obtained some good results. But their methods did not have high convergence order.

In this paper, we set up a class of high algebraic accuracy numerical integration methods which are proposed by compositing the two-point and three-point Gauss-Legendre formulas. We design these formulas to calculate integration of fuzzy functions. We also present the methods' remainder terms and give corresponding convergence theorems. Compared with some approaches for approximating fuzzy integrations before, our methods are superior to those formulas on both amount of calculation and quadrature error. The structure of this paper is as follows.

In Section 2, we recall some basic definitions and results on integration of fuzzy functions. In Section 3, we introduce the two-point and three-point Gauss-Legendre formulas and their composite method. Then we design them to solve fuzzy integration. We also put up methods' reminder term representations and convergence theorems. The proposed algorithms are illustrated by solving two examples in Section 4 and the conclusion is drawn in Section 5.

\section{Preliminaries}

2.1. Integration of Fuzzy Function. Let $E^{1}$ be the set of all real fuzzy numbers which are normal, upper semicontinuous, convex, and compactly supported fuzzy sets. 
Definition 1 (see [12]). A fuzzy number $u$ in parametric form is a pair $(\underline{u}, \bar{u})$ of functions $\underline{u}(r), \bar{u}(r), 0 \leq r \leq 1$, which satisfies the following requirements:

(1) $\underline{u}(r)$ is a bounded monotonic increasing left continuous function,

(2) $\bar{u}(r)$ is a bounded monotonic decreasing left continuous function,

(3) $\underline{u}(r) \leq \bar{u}(r), 0 \leq r \leq 1$.

Based on Zadeh's principle of extension, Goetschel et al. [13] presented fuzzy numbers' addiction and multiplication by $k$ which are as follows. Let $x=(\underline{x}(r), \bar{x}(r)), y=$ $(y(r), \bar{y}(r)) \in E^{1}, 0 \leq r \leq 1$, and real number $k$, as follows:

(1) $x+y=(\underline{x}(r)+\underline{y}(r), \bar{x}(r)+\bar{y}(r))$,

(2) $x-y=(\underline{x}(r)-\bar{y}(r), \bar{x}(r)-\underline{y}(r))$,

(3) $k x=\left\{\begin{array}{l}(k x(r), k \bar{x}(r)), k \geq 0, \\ (k \overline{\bar{x}}(r), k x(r)), k<0 .\end{array}\right.$

For arbitrary fuzzy numbers $x=(\underline{x}(r), \bar{x}(r)), y=$ $(y(r), \bar{y}(r)) \in E^{1}$, the quantity

$$
D(x, y)=\sup _{0 \leq r \leq 1}\{\max [|\underline{x}(r)-\underline{y}(r)|,|\bar{x}(r)-\bar{y}(r)|]\}
$$

is the distance between $x$ and $y$. A function $f: R^{1} \rightarrow E^{1}$ is called a fuzzy function. If for arbitrary fixed $t_{0} \in R^{1}$ and $\epsilon>0$, a $\delta>0$ such that

$$
\left|t-t_{0}\right|<\delta \Longrightarrow D\left[f(t), f\left(t_{0}\right)\right]<\epsilon,
$$

exists, $f$ is said to be continuous.

Definition 2 (see [14]). Assume $f:[a, b] \rightarrow E^{1}$. For each partition $P=\left\{t_{0}, t_{1}, \ldots, t_{n}\right\}$ of $[a, b]$ and for arbitrary $\xi_{i}$ : $t_{i-1} \leq \xi_{i} \leq t_{i}, 1 \leq i \leq n$, let

$$
R_{p}=\sum_{i=1}^{n} f\left(\xi_{i}\right)\left(t_{i}-t_{i-1}\right)
$$

The definite integral of $f(t)$ over $[a, b]$ is

$$
\int_{a}^{b} f(t) d t=\lim R_{p}, \quad \max _{1 \leq i \leq n}\left|t_{i}-t_{i-1}\right| \longrightarrow 0,
$$

provided that this limit exists in the metric $D$. If the fuzzy function $f(t)$ is continuous in the metric $D$, its definite integral exists. Furthermore,

$$
\begin{aligned}
& \left(\underline{\left.\int_{a}^{b} f(t ; r) d t\right)}=\int_{a}^{b} f(t ; r) d t,\right. \\
& \left(\overline{\left.\int_{a}^{b} f(t ; r) d t\right)}=\int_{a}^{b} \bar{f}(t ; r) d t .\right.
\end{aligned}
$$

It should be noted that the fuzzy integral also can be defined using the Lebesgue-type approach $[15,16]$. More details about properties of fuzzy integral are given in [17].
2.2. Gauss-Legendre Formulas. Gauss quadrature formula is the highest algebraic accuracy of interpolation quadrature formula. By reasonably selecting quadrature nodes and quadrature coefficients of the form of

$$
\int_{a}^{b} f(x) d x \approx \sum_{k=0}^{n} A_{k} f\left(x_{k}\right)
$$

we can obtain the interpolation quadrature formula with the highest algebraic accuracy; that is, $2 n+1$. Using root nodes of $n+1$ order Legendre orthogonal polynomial on special interval $[-1,1]$, we can propose Gauss-Legendre quadrature formula (7).

Lemma 3 (see [6]). The reminder term of Gauss-Legendre quadrature formula (7) is

$$
\begin{aligned}
E(f) & =\int_{a}^{b} f(x) d x-\sum_{k=0}^{n} A_{k} f\left(x_{k}\right) \\
& =\int_{a}^{b} \frac{f^{(2 n+2)}(\xi)}{(2 n+2) !} \omega^{2}(x) d x, \quad \xi \in[a, b],
\end{aligned}
$$

where $\omega(x)=\prod_{i=0}^{n}\left(x-x_{i}\right)$ and $x_{i}$ are Gauss nodes.

In particular, when $n=1,(7)$ is two-point Gauss-Legendre quadrature formula:

$$
\int_{a}^{b} f(x) d x \approx \frac{b-a}{2}\left(f\left(x\left(-\frac{\sqrt{3}}{3}\right)\right)+f\left(x\left(\frac{\sqrt{3}}{3}\right)\right)\right),
$$

where $x(t)=(a+b) / 2+((b-a) / 2) t$ and its reminder term is

$$
\begin{array}{r}
E(f)=\int_{a}^{b} \frac{f^{(4)}(\xi)}{4 !}\left(x-x\left(-\frac{\sqrt{3}}{3}\right)\right)^{2}\left(x-x\left(\frac{\sqrt{3}}{3}\right)\right)^{2} d x \\
\xi \in[a, b] .
\end{array}
$$

When $n=2,(7)$ is three-point Gauss-Legendre quadrature formula:

$$
\begin{aligned}
\int_{a}^{b} f(x) d x \approx \frac{b-a}{2} & \left(\frac{5}{9} f\left(x\left(-\frac{\sqrt{15}}{5}\right)\right)\right. \\
& \left.+\frac{8}{9} f\left(\frac{a+b}{2}\right)+\frac{5}{9} f\left(x\left(\frac{\sqrt{15}}{5}\right)\right)\right),
\end{aligned}
$$

and its reminder term is

$$
\begin{aligned}
& E(f)=\int_{a}^{b} \frac{f^{(6)}(\xi)}{6 !}\left(x-x\left(-\frac{\sqrt{15}}{5}\right)\right)^{2} \\
& \times\left(x-\frac{a+b}{2}\right)^{2}\left(x-x\left(\frac{\sqrt{15}}{5}\right)\right)^{2} d x, \\
& \xi \in[a, b] .
\end{aligned}
$$




\subsection{Convergence Order of Composite Method}

Definition 4 (see [18]). Suppose $I=\int_{a}^{b} f(x) d x$, and $I_{n}$ is a composite numerical integration method. If $h \rightarrow 0$, it satisfies

$$
\lim _{h \rightarrow 0} \frac{I-I_{n}}{h^{p}}=C, \quad C \neq 0,
$$

and we call $I_{n}$ a $p$ order convergent method.

For instance, composite Trapezoid method

$$
\int_{a}^{b} f(x) d x \approx \frac{b-a}{2} \sum_{k=0}^{n-1}\left(f\left(x_{i}\right)+f\left(x_{i+1}\right)\right)
$$

and composite Simpson

$$
\int_{a}^{b} f(x) d x \approx \frac{h}{6} \sum_{k=0}^{n-1}\left(f\left(x_{i}\right)+4 f\left(x_{i+1 / 2}\right)+f\left(x_{i+1}\right)\right)
$$

have two-order and four-order convergence property, respectively.

\section{Gauss-Legendre Formulas for Solving Fuzzy Integral}

\subsection{Composite Formulas and Their Error Reminders}

Theorem 5. Let $f(x) \in C^{(6)}[a, b], x_{k}=x_{0}+k h, k=0,1$, $2, \ldots, n, h=(b-a) / n$; then the reminder term of composite three-point formulas Gauss-Legendre

$$
\begin{array}{r}
\int_{a}^{b} f(x) d x \approx \frac{h}{2} \sum_{i=0}^{n-1}\left(\frac{5}{9} f\left(x_{i+1 / 2}-\frac{\sqrt{15} h}{10}\right)+\frac{8}{9} f\left(x_{i+1 / 2}\right)\right. \\
\left.+\frac{5}{9} f\left(x_{i+1 / 2}+\frac{\sqrt{15} h}{10}\right)\right)
\end{array}
$$

is

$$
E(f(x))=\frac{(b-a) h^{6}}{2^{6} \times 31500} f^{(6)}(\xi), \quad \xi \in[a, b],
$$

and it has six-order convergence property.

Proof. We first consider the remainder term of three-point Gauss-Legendre. By Lemma 3,

$$
\begin{aligned}
E(g) & =\int_{-1}^{1} g(t) d t-\left(\frac{5}{9} g\left(-\frac{\sqrt{15}}{5}\right)+\frac{8}{9} g(0)+\frac{5}{9} g\left(\frac{\sqrt{15}}{5}\right)\right) \\
& =c g^{(6)}(\xi), \quad \xi \in[-1,1] .
\end{aligned}
$$

Let $g(t)=t^{6}$, and

$$
\begin{gathered}
g^{(6)}(t) \equiv 720, \quad \int_{-1}^{1} t^{6} d t=\frac{2}{7}, \\
I\left(t^{6}\right)=\frac{5}{9}\left(-\frac{\sqrt{15}}{5}\right)^{6}+\frac{5}{9}\left(-\frac{\sqrt{15}}{5}\right)^{6}=\frac{6}{25} .
\end{gathered}
$$

We have

$$
E\left(t^{6}\right)=\int_{-1}^{1} t^{6} d t-I\left(t^{6}\right)=\frac{2}{7}-\frac{6}{25}=720 c
$$

and $c=1 / 15750$. Thus, we obtain the reminder term of threepoint formulas Gauss-Legendre:

$$
E(g)=\frac{1}{15750} g^{(6)}(\xi), \quad \xi \in[-1,1]
$$

Now we study the integral remainder term of composite three-point Gauss-Legendre:

$$
\int_{a}^{b} f(x) d x=\sum_{i=0}^{n-1} \int_{x_{i}}^{x_{i+1}} f(x) d x
$$

where $P=\left\{x_{0}, x_{1}, \ldots, x_{n}\right\}$ is about equidistant partition on $[a, b]$. Let $x(t)=\left(x_{i}+x_{i+1}\right) / 2+(h / 2) t, x_{i+1 / 2}=\left(x_{i}+x_{i+1}\right) / 2$; then

$$
\int_{x_{i}}^{x_{i+1}} f(x) d x=\frac{h}{2} \int_{-1}^{1} f\left(x_{i+1 / 2}+\frac{h}{2} t\right) d t .
$$

By means of three-point Gauss-Legendre formula and its reminder term (21), we have

$$
\begin{aligned}
\int_{a}^{b} f(x) d x= & \sum_{i=0}^{n-1} \frac{h}{2} \int_{-1}^{1} f\left(x_{i+1 / 2}+\frac{h}{2} t\right) d t \\
= & \frac{h^{n-1}}{2} \sum_{i=0}^{n}\left(\frac{5}{9} f\left(x_{i+1 / 2}-\frac{\sqrt{3} h}{2 \sqrt{5}}\right)+\frac{8}{9} f\left(x_{i+1 / 2}\right)\right. \\
& +\frac{5}{9} f\left(x_{i+1 / 2}+\frac{\sqrt{3} h}{2 \sqrt{5}}\right) \\
& \left.+\frac{h^{6}}{2^{6} \times 15750} f^{(6)}\left(\xi_{i}\right)\right), \\
= & \frac{h^{n-1}}{2} \sum_{i=0}^{n}\left(\frac{5}{9} f\left(x_{i+1 / 2}-\frac{\sqrt{3} h}{2 \sqrt{5}}\right)+\frac{8}{9} f\left(x_{i+1 / 2}\right)\right. \\
& +\frac{\left.x_{i+1}\right]}{2} \sum_{i=0}^{n-1}\left(\frac{5}{9} f\left(x_{i+1 / 2}-\frac{\sqrt{3} h}{2 \sqrt{5}}\right)+\frac{8}{9} f\left(x_{i+1 / 2}\right)\right. \\
& \left.+\frac{h^{7}}{2^{7}} \sum_{i=0}^{n-1} \frac{1}{15750} f\left(x_{i+1 / 2}+\frac{\sqrt{3} h}{2 \sqrt{5}}\right)\right) \\
\left(\xi_{i}\right), & \frac{5}{9} f\left(x_{i+1 / 2}+\frac{\xi^{6}}{2}\right)
\end{aligned}
$$


So we get the reminder term of composite three-point GaussLegendre as follows:

$$
E(f(x))=\frac{(b-a) h^{6}}{2^{6} \times 31500} f^{(6)}(\xi), \quad \xi \in[a, b] .
$$

Since

$$
\lim _{h \rightarrow 0} \frac{I-I_{n}}{h^{6}}=\frac{(b-a)}{2^{6} \times 31500} f^{(6)}(\xi),
$$

we know it has six-order convergence property.

In similar way, we obtain the reminder term of composite two-point formulas Gauss-Legendre as follows.

Theorem 6. Let $f(x) \in C^{(4)}[a, b], x_{k}=x_{0}+k h, k=$ $0,1,2, \ldots, n, h=(b-a) / n$; then the reminder term of composite two-point formulas Gauss-Legendre

$$
\begin{aligned}
& \int_{a}^{b} f(x) d x \\
& \quad \approx \frac{h}{2} \sum_{k=0}^{n-1}\left(f\left(x_{i+1 / 2}-\frac{\sqrt{3} h}{6}\right)+f\left(x_{i+1 / 2}+\frac{\sqrt{3} h}{6}\right)\right),
\end{aligned}
$$

is

$$
E(f(x))=\frac{(b-a) h^{4}}{2^{4} \times 270} f^{(4)}(\xi), \quad \xi \in[a, b],
$$

and it has four-order convergence property.

3.2. Gauss-Legendre Formulas to Fuzzy Integration. In this subsection, we apply composite Gauss-Legendre formulas to solve fuzzy integration and give their reminder terms and convergence theorems.

Applying formulas (16) and (27) to numerical integration for fuzzy function (13), we have

$$
\begin{aligned}
& \int_{a}^{b} f(x ; r) d x \approx \frac{h}{2} \sum_{i=0}^{n-1}\left[\frac{5}{9} f\left(x_{i+1 / 2}-\frac{\sqrt{15} h}{10} ; r\right)\right. \\
& +\frac{8}{9} \frac{f}{}\left(x_{i+1 / 2} ; r\right) \\
& \left.+\frac{5}{9} f\left(x_{i+1 / 2}+\frac{\sqrt{15} h}{10} ; r\right)\right], \\
& \int_{a}^{b} \bar{f}(x ; r) d x \approx \frac{h}{2} \sum_{i=0}^{n-1}\left[\frac{5}{9} \bar{f}\left(x_{i+1 / 2}-\frac{\sqrt{15} h}{10} ; r\right)\right. \\
& +\frac{8}{9} f\left(x_{i+1 / 2} ; r\right) \\
& \left.+\frac{5}{9} f\left(x_{i+1 / 2}+\frac{\sqrt{15} h}{10} ; r\right)\right] \text {, } \\
& 0 \leq r \leq 1
\end{aligned}
$$

$$
\begin{array}{r}
\int_{a}^{b} \underline{f}(x ; r) d x \approx \frac{h}{2} \sum_{k=0}^{n-1}\left[\underline{f}\left(x_{i+1 / 2}-\frac{\sqrt{3} h}{6} ; r\right)\right. \\
\left.+\underline{f}\left(x_{i+1 / 2}+\frac{\sqrt{3} h}{6} ; r\right)\right], \\
\int_{a}^{b} \bar{f}(x ; r) d x \approx \frac{h}{2} \sum_{k=0}^{n-1}\left[\bar{f}\left(x_{i+1 / 2}-\frac{\sqrt{3} h}{6} ; r\right)\right. \\
\left.+\bar{f}\left(x_{i+1 / 2}+\frac{\sqrt{3} h}{6} ; r\right)\right], \\
0 \leq r \leq 1 .
\end{array}
$$

Theorem 7. Suppose $f(x ; r) \in C^{6}[a, b]$ about $x$ and $f^{(6)}(x ; r) \geq 0, \bar{f}^{(6)}(x ; r) \geq 0,0 \leq r \leq 1$; then the reminder terms of composite three-point Gauss-Legendre formulas (29) for fuzzy integration are

$$
\begin{aligned}
& E(\underline{f}(x ; r))=\frac{(b-a) h^{6}}{2^{6} \times 31500} \underline{f}^{(6)}(\underline{\xi} ; r), \\
& E(\bar{f}(x: r))=\frac{(b-a) h^{6}}{2^{6} \times 31500} \bar{f}^{(6)}(\bar{\xi}: r),
\end{aligned}
$$

where $\underline{\xi}, \bar{\xi} \in[a, b], 0 \leq r \leq 1$.

Proof. From formulas (16) and (17), we get

$$
\begin{aligned}
\int_{a}^{b} f(x) d x= & \frac{h^{n-1}}{2} \sum_{i=0}\left[\frac{5}{9} f\left(x_{i+1 / 2}-\frac{\sqrt{15} h}{10}\right)+\frac{8}{9} f\left(x_{i+1 / 2}\right)\right. \\
& \left.+\frac{5}{9} f\left(x_{i+1 / 2}+\frac{\sqrt{15} h}{10}\right)\right] \\
& +\frac{(b-a) h^{6}}{2^{6} \times 31500} f^{(6)}(\xi), \quad \xi \in[a, b] .
\end{aligned}
$$

Using the above formula for fuzzy integration in parametric form, we have

$$
\begin{aligned}
& \int_{a}^{b} f(x ; r) d x= \frac{h^{h-1}}{2} \sum_{i=0}^{n-0}\left[\frac{5}{9} \underline{f}\left(x_{i+1 / 2}-\frac{\sqrt{15} h}{10} ; r\right)\right. \\
&+\frac{8}{9} \underline{f}\left(x_{i+1 / 2} ; r\right) \\
&\left.+\frac{5}{9} f\left(x_{i+1 / 2}+\frac{\sqrt{15} h}{10} ; r\right)\right] \\
&+ \frac{(b-a) h^{6}}{2^{6} \times 31500} f^{(6)}(\underline{\xi} ; r), \\
& \int_{a}^{b} \bar{f}(x ; r) d x=\frac{h^{n}}{2} \sum_{i=0}^{n-1}\left[\frac{5}{9} \bar{f}\left(x_{i+1 / 2}-\frac{\sqrt{15} h}{10} ; r\right)\right. \\
&+\frac{8}{9} f\left(x_{i+1 / 2} ; r\right)
\end{aligned}
$$




$$
\begin{aligned}
& \left.+\frac{5}{9} f\left(x_{i+1 / 2}+\frac{\sqrt{15} h}{10} ; r\right)\right] \\
& +\frac{(b-a) h^{6}}{2^{6} \times 31500} \bar{f}^{(6)}(\bar{\xi}: r),
\end{aligned}
$$

where $\xi, \bar{\xi} \in[a, b], 0 \leq r \leq 1$.

So the reminder terms of composite three-point GaussLegendre formulas (29) for fuzzy integration are (31).

Theorem 8. Let $f(x ; r) \in C^{6}[a, b]$ about $x, 0 \leq r \leq 1$; then

$$
\begin{gathered}
\lim _{h \rightarrow 0}\left[\frac { h } { 2 } \sum _ { i = 0 } ^ { n - 1 } \left(\frac{5}{9} f\left(x_{i+1 / 2}-\frac{\sqrt{15} h}{10} ; r\right)+\frac{8}{9} f\left(x_{i+1 / 2} ; r\right)\right.\right. \\
\left.\left.\quad+\frac{5}{9} \frac{f}{-}\left(x_{i+1 / 2}+\frac{\sqrt{15} h}{10} ; r\right)\right)\right] \\
=\int_{a}^{b} \frac{f}{b}(x ; r) d x, \\
\lim _{h \rightarrow 0}\left[\frac { h ^ { n - 1 } } { 2 } \sum _ { i = 0 } ^ { n - 1 } \left(\frac{5}{9} \bar{f}\left(x_{i+1 / 2}-\frac{\sqrt{15} h}{10} ; r\right)+\frac{8}{9} f\left(x_{i+1 / 2} ; r\right)\right.\right. \\
\left.\left.\quad+\frac{5}{9} f\left(x_{i+1 / 2}+\frac{\sqrt{15} h}{10} ; r\right)\right)\right] \\
=\int_{a}^{b} \bar{f}(x ; r) d x .
\end{gathered}
$$

Proof. From Theorem 7, we have

$$
\begin{aligned}
E(\underline{f}(x ; r))= & \int_{a}^{b} \underline{f}(x ; r) d x \\
& -\frac{h^{n-1}}{2} \sum_{i=0}\left(\frac{5}{9} \underline{f}\left(x_{i+1 / 2}-\frac{\sqrt{15} h}{10} ; r\right)\right. \\
& +\frac{8}{9} \underline{f}\left(x_{i+1 / 2} ; r\right) \\
& \left.+\frac{5}{9} \frac{f}{-}\left(x_{i+1 / 2}+\frac{\sqrt{15} h}{10} ; r\right)\right) \\
E(\bar{f}(x: r))= & \frac{(b-a) h^{6}}{2^{6} \times 31500} \frac{f^{(6)}}{f}(\underline{\xi} ; r), \\
& -\frac{h^{n-1}}{2} \sum_{i=0}^{b}\left(\frac{5}{9} \bar{f}\left(x_{i+1 / 2}-\frac{\sqrt{15} h}{10} ; r\right)\right. \\
& +\frac{8}{9} f\left(x_{i+1 / 2} ; r\right)
\end{aligned}
$$

$$
\begin{gathered}
\left.+\frac{5}{9} f\left(x_{i+1 / 2}+\frac{\sqrt{15} h}{10} ; r\right)\right) \\
=\frac{(b-a) h^{6}}{2^{6} \times 31500} \bar{f}^{(6)}(\bar{\xi}: r),
\end{gathered}
$$

where $\underline{\xi}, \bar{\xi} \in[a, b], 0 \leq r \leq 1$.

Since $f^{(6)}(x ; r), \bar{f}^{(6)}(x ; r)$ are bounded over $[a, b]$, we easily get the following fact:

$$
E(\underline{f} ; r) \longrightarrow 0, \quad E(\bar{f} ; r) \longrightarrow 0,
$$

if $h \rightarrow 0$.

Similarly, we have the following convergence theorem.

Theorem 9. Suppose $f(x ; r) \in C^{4}[a, b]$ about $x$ and $f^{(4)}(x ; r) \geq 0, \bar{f}^{(4)}(x ; r) \geq 0,0 \leq r \leq 1$; then the reminder terms of composite two-point Gauss-Legendre formulas (30) for fuzzy integration are

$$
\begin{gathered}
E(\underline{f}(x ; r))=\frac{(b-a) h^{4}}{2^{4} \times 270} \underline{f}^{(4)}(\underline{\xi} ; r), \\
E(\bar{f}(x: r))=\frac{(b-a) h^{4}}{2^{4} \times 270} \bar{f}^{(4)}(\bar{\xi}: r),
\end{gathered}
$$

where $\xi, \bar{\xi} \in[a, b], 0 \leq r \leq 1$. And

$$
\begin{aligned}
\lim _{h \rightarrow 0} \frac{h}{2} \sum_{k=0}^{n-1}\left[\underline{f}\left(x_{i+1 / 2}-\frac{\sqrt{3} h}{6} ; r\right)+\underline{f}\left(x_{i+1 / 2}+\frac{\sqrt{3} h}{6} ; r\right)\right] \\
\quad=\int_{a}^{b} \underline{f}(x ; r) d x, \\
\lim _{h \rightarrow 0} \frac{h}{2} \sum_{k=0}^{n-1}\left[\bar{f}\left(x_{i+1 / 2}-\frac{\sqrt{3} h}{6} ; r\right)+\bar{f}\left(x_{i+1 / 2}+\frac{\sqrt{3} h}{6} ; r\right)\right] \\
\quad=\int_{a}^{b} \bar{f}(x ; r) d x .
\end{aligned}
$$

\section{Numerical Examples}

Example 1. Consider the following fuzzy integral:

$$
\int_{0}^{2} \tilde{k} x^{4} d x, \quad \tilde{k}=(r, 2-r) .
$$

The exact solution is $(32 / 5)(r, 2-r)=6.400000(r, 2-r)$.

From the two-point Gauss-Legendre formula:

$$
\begin{array}{lll}
Q(\underline{f} ; r)=6.222222 r, & Q(\bar{f} ; r)=6.222222(2-r), \\
E(\underline{f} ; r)=0.177778 r, & E(\bar{f} ; r)=0.177778(2-r),
\end{array}
$$

it is clear that formula (37) holds. 
TABLE 1: Numerical solutions and errors between different methods $(h=1)$.

\begin{tabular}{lcc}
\hline Methods & Numerical solution & Error \\
\hline Trapezoidal formula & $0.68393972(-1+r, 1-r)$ & 0.05181917 \\
Simpson formula & $0.63233368(-1+r, 1-r)$ & 0.00021303 \\
Two-point Gauss-L & $0.63197875(-1+r, 1-r)$ & 0.00014180 \\
Three-point Gauss-L & $0.63212025(-1+r, 1-r)$ & 0.00000040 \\
\hline
\end{tabular}

By the two-point Gauss-Legendre formula with $h=1$

$$
\begin{array}{ll}
Q(\underline{f} ; r)=6.388888 r, & Q(\bar{f} ; r)=6.388888(2-r), \\
E(\underline{f} ; r)=0.011112 r, & E(\bar{f} ; r)=0.011112(2-r),
\end{array}
$$

it is clear that formula (37) holds. Now with $h=1 / 2$,

$$
\begin{array}{ll}
Q(\underline{f} ; r)=6.399305 r, & Q(\bar{f} ; r)=6.399305(2-r), \\
E(\underline{f} ; r)=0.000695 r, & E(\bar{f} ; r)=0.000695(2-r) .
\end{array}
$$

Equation (38) holds too.

Example 2. Consider the following fuzzy integral:

$$
\int_{0}^{1} \tilde{k} e^{-x} d x, \quad \tilde{k}=(-1+r, 1-r)
$$

The exact solution is $(1-1 / e)(-1+r, 1-r)=$ $0.63212055 \cdots(-1+r, 1-r)$.

We calculate numerically the above integral using Trapezoidal formula, Simpson formula, composite two-point Gauss-Legendre, and three-point Gauss-Legendre methods with $h=1, h=1 / 2$, and $h=1 / 4$. Some comparisons about the numerical solutions and the errors between the different methods are shown in Tables 1, 2, and 3. All data are denoted with eight-bit significant digits and errors are calculated by the distance between exact solution and numerical solution.

From the above tables' figures, we can clearly see that our methods have better approximation than the Trapezoidal formula and Simpson formula on the same fuzzy integration, in which the composite three-point Gauss-Legendre is really the case.

\section{Conclusion}

In this work, we applied composite Gauss-Legendre formulas to solve fuzzy integral over a finite interval $[a, b]$. Since this integration yields fuzzy number in parametric form, we use the parametric form of the methods. The integration of triangular fuzzy number is a triangular fuzzy number. Numerical examples showed that our methods are practical and efficient while computing fuzzy integral on a larger interval $[a, b]$.
TABLE 2: Numerical solutions and errors between different methods $(h=0.5)$.

\begin{tabular}{lcc}
\hline Methods & Numerical solution & Error \\
\hline Trapezoidal formula & $0.64523519(-1+r, 1-r)$ & 0.01311464 \\
Simpson formula & $0.63213418(-1+r, 1-r)$ & 0.00001363 \\
Two-point Gauss-L & $0.63211148(-1+r, 1-r)$ & 0.00000877 \\
Three-point Gauss-L & $0.63212055(-1+r, 1-r)$ & 0.00000000 \\
\hline
\end{tabular}

TABLE 3: Numerical solutions and errors between different methods $(h=0.25)$.

\begin{tabular}{lcc}
\hline Methods & Numerical solution & Error \\
\hline Trapezoidal formula & $0.63540943(-1+r, 1-r)$ & 0.00328888 \\
Simpson formula & $0.63212141(-1+r, 1-r)$ & 0.00000086 \\
Two-point Gauss-L & $0.63211998(-1+r, 1-r)$ & 0.00000057 \\
Three-point Gauss-L & $0.63212055(-1+r, 1-r)$ & 0.00000000 \\
\hline
\end{tabular}

\section{Conflict of Interests}

The authors declare that there is no conflict of interests regarding the publication of this paper.

\section{Acknowledgments}

The work is supported by the Natural Scientific Funds of China (nos. 61262022 and 21175108) and the Youth Research Ability Project of Northwest Normal University (NWNULKQN-1120).

\section{References}

[1] P. J. Davis and P. Rabinowitz, Methods of Numerical Integration, Academic Press, Orlando, Fla, USA, 2nd edition, 1984.

[2] R. L. Burden and J. Douglas, Numerical Analyisis, Thomson Learning, Boston, Mass, USA, 2001.

[3] B. Q. Liu, "Asymptotic analysis for some numerical integral formulas," Communication on Applied Mathematics and Computation, vol. 14, no. 2, pp. 83-87, 2000.

[4] B. Q. Liu, "Limit properties about a class of Gaussian integration formulas," Journal of Engineering Mathematics, vol. 20, no. 4, pp. 137-139, 2003.

[5] S. F. Qiu and Z. W. Wang, "Asymptotic properties of the intermediate point to numerical integration and its Applications," Mathematics in Practice and Theory, vol. 36, no. 5, pp. 218-223, 2006.

[6] Q. H. Zhao, "Correction formulas for numerical integral," Mathematics in Practice and Theory, vol. 37, pp. 207-208, 2007.

[7] H. J. Zimmerman, "Fuzzy sets theory and its applications," Fuzzy Sets and Systems, vol. 24, pp. 319-330, 1987.

[8] T. Allahviranloo, "Newton Cot's methods for integration of fuzzy functions," Applied Mathematics and Computation, vol. 166, no. 2, pp. 339-348, 2005.

[9] T. Allahviranloo, "Romberg integration for fuzzy function," Applied Mathematics and Computation, vol. 168, pp. 886-876, 2005.

[10] T. Allahviranloo and M. Otadi, "Gaussian quadratures for approximate of fuzzy integrals," Applied Mathematics and Computation, vol. 170, no. 2, pp. 874-885, 2005. 
[11] T. Allahviranloo and M. Otadi, "Gaussian quadratures for approximate of fuzzy multiple integrals," Applied Mathematics and Computation, vol. 172, no. 1, pp. 175-187, 2006.

[12] C. X. Wu and M. Ma, "On emdedding problem of fuzzy number space-part I," Fuzzy Sets and Systems, vol. 44, no. 1, pp. 33-38, 1991.

[13] R. Goetschel, Jr. and W. Voxman, "Elementary calculus," Fuzzy Sets and Systems, vol. 18, no. 1, pp. 31-43, 1986.

[14] M. L. Puri and D. A. Ralescu, "Fuzzy random variables," Journal of Mathematical Analysis and Applications, vol. 114, no. 2, pp. 409-422, 1986.

[15] O. Kaleva, "Fuzzy differential equations," Fuzzy Sets and Systems, vol. 24, no. 3, pp. 301-317, 1987.

[16] M. Matłoka, "On fuzzy integrals," in Proceedings of the 2nd Polish Symposium on Interval and Fuzzy Mathematics, vol. 18, pp. 167-170, Plite Chnick Poznansk, 1987.

[17] J. Stoer and R. Bulirsch, Introduction to Numerical Analysis, Springer, New York, NY, USA, 1980.

[18] Q. Y. Li, N. C. Wang, and D. Y. Yi, Numerical Analyisis, Tsuing University Press, Springer, 2003. 


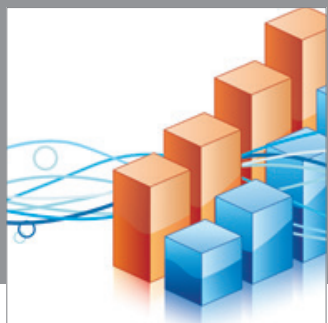

Advances in

Operations Research

mansans

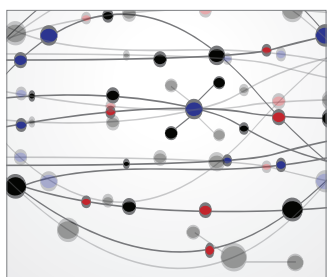

The Scientific World Journal
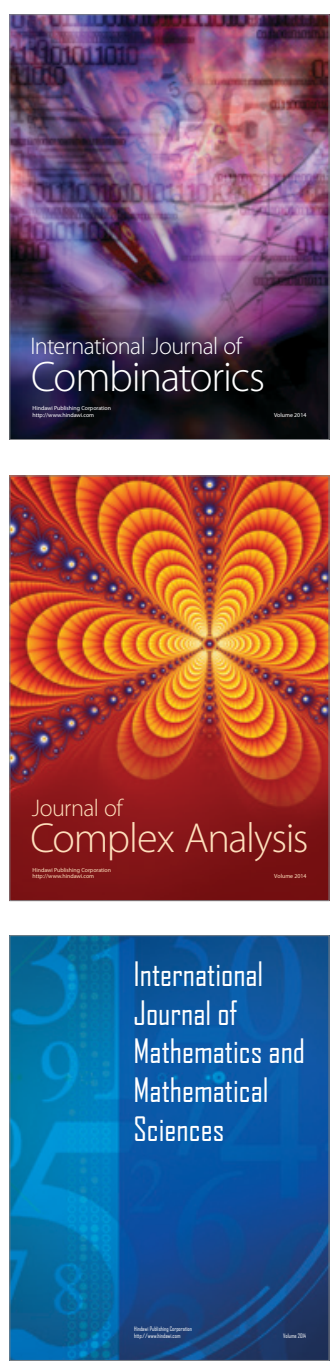
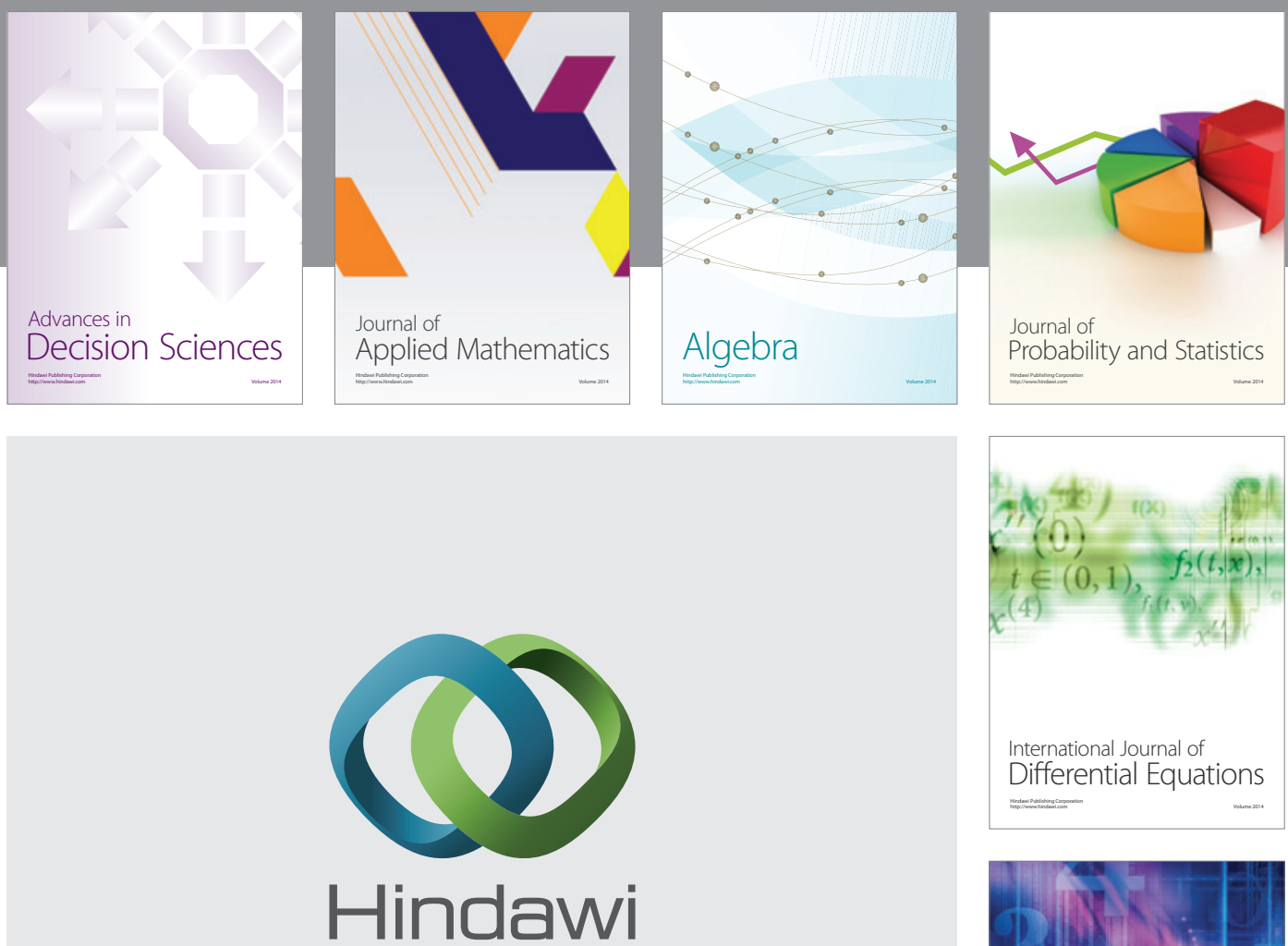

Submit your manuscripts at http://www.hindawi.com
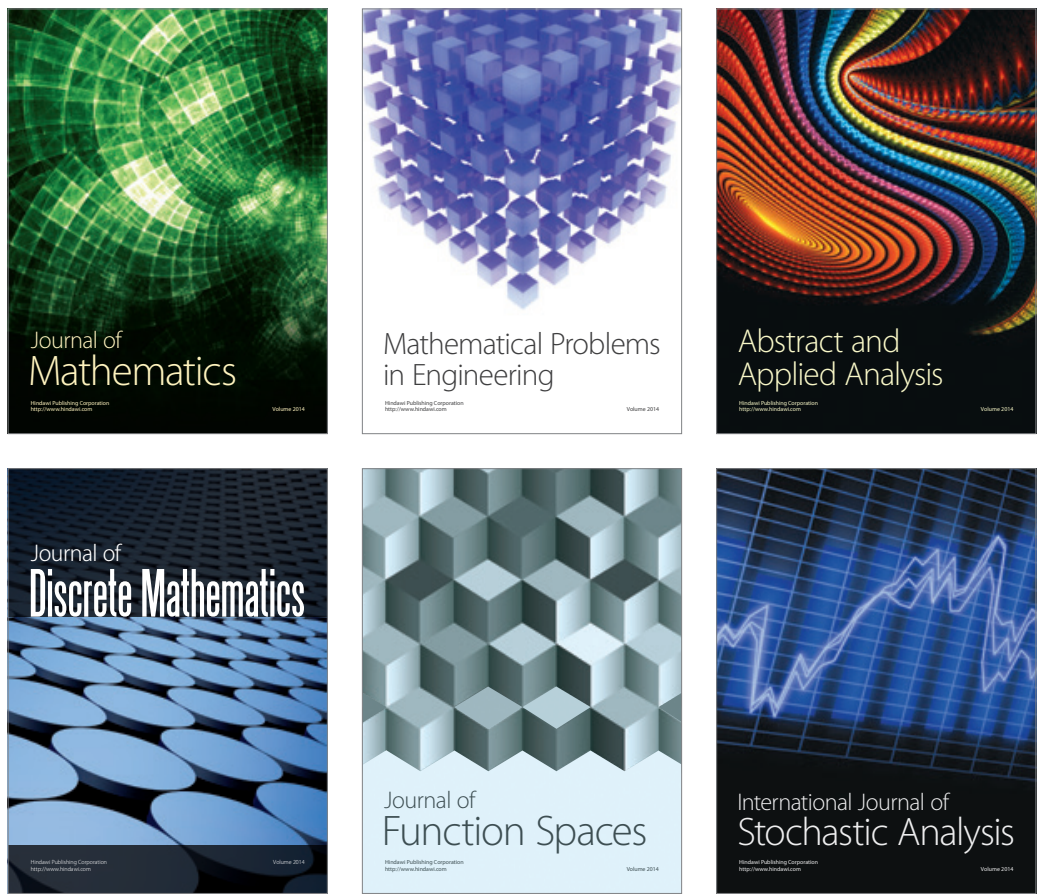

Journal of

Function Spaces

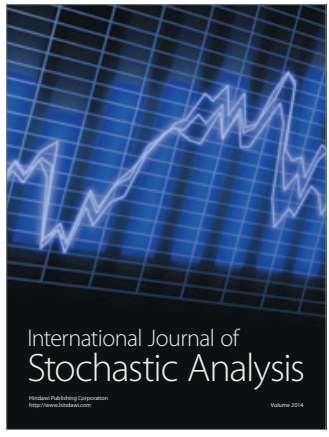

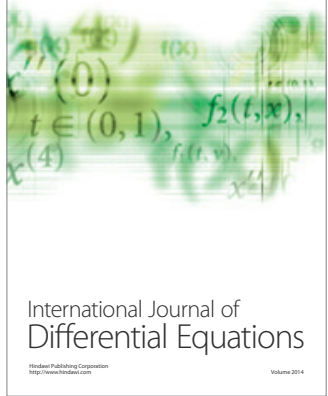
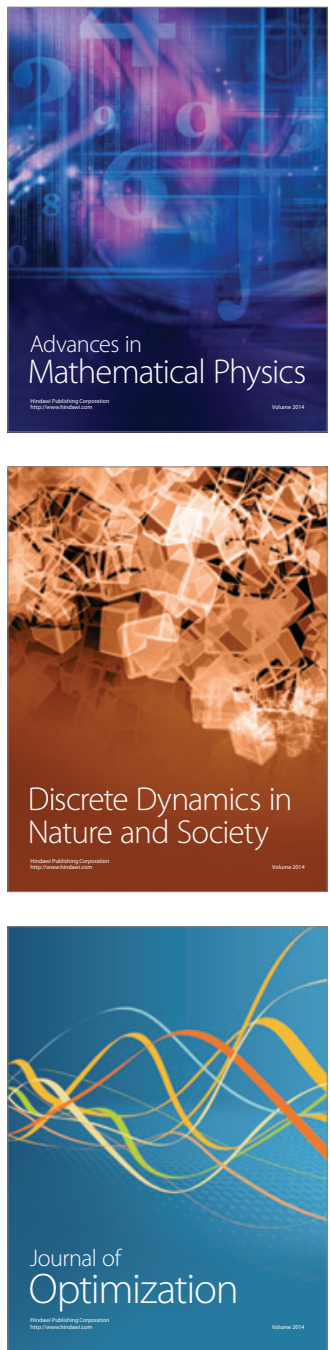